\title{
Homology Modeling and characterization of Phosphoenolpyruvate Carboxykinase (PEPCK) from Schistosoma japonicum
}

\author{
Akalesh Kumar Verma* ${ }^{1}$, Purobi Nath ${ }^{2}$, Surya Bali Prasad ${ }^{1}$, Devid Kardong ${ }^{3}$, \\ Jashodeb Arjun $^{4}$, Durba Kashyap ${ }^{4}$ and Ramesh Chillawar ${ }^{5}$ \\ ${ }^{I}$ Cell and Tumor Biology Laboratory, Department of Zoology, North-Eastern Hill University, \\ Shillong-793022, India. \\ ${ }^{2}$ Parasitology \& Ethnopharmacology Laboratory, Department of Zoology, North-Eastern Hill University, \\ Shillong-793022, India. \\ ${ }^{3}$ Depment of Life Sciences, Dibrugarh University, Dibrugarh- 786004, India. \\ ${ }^{4}$ Department of Zoology, Biochemistry Division, Lumding College, Lumding-782447, Assam, India. \\ ${ }^{5}$ Department of Botany, Yeshwant Mahavidalaya Nanded (Maharashtra) - 431602, India.
}

\begin{abstract}
Phosphoenolpyruvate carboxykinase (PEPCK), a carboxylase enzyme is present in all living organisms. It catalyzes metal-nucleotide coupled reversible decarboxylation and phosphorylation between phosphoenolpyruvate (PEP) and oxaloacetate (OAA) depending on the system and the availability of the intermediates. In fungi, plants and in most bacteria, production of PEP from OAA by PEPCK is the key step during gluconeogenesis. In healthy human cytosolic PEPCK enzyme is present only during glucose starvation; cytosolic PEPCK rapidly disappears on the replenishment of glucose due to hormonal control of the transcription of the cytosolic PEPCK- gene. In some parasitic helminthes like Ascaris suum, nematodes such as Haemonchus contortus, PEPCK carry out the reverse reaction to produce OAA from PEP. In Trypanosoma cruzi and all species of the genus Leishmania, this enzyme is very active even in the presence of high level of carbohydrate. There is a significant functional difference between parasitic PEPCK and mammalian host PEPCK. These differences between the mammalian host and the parasite enzyme strongly support the belief that PEPCK should be further investigated as a possible target for selective chemotherapeutic agents. The experimental $3 D$ structure of PEPCK of Schistosoma japonicum is not available in protein data bank. Therefore, based on the knowledge of the best template (3DT7), model of 3D structure of Schistosoma japonicum PEPCK was prepared using Modeller v9.10 software and processed in to energy minimization, Ramachandran plot analysis, quality assessment and characterization.
\end{abstract}

Keywords: Antihelminthic, Docking, Homology modeling, Schistosoma japonicum, Phosphoenolpyruvate carboxykinase

\section{INTRODUCTION}

Phosphoenolpyruvate carboxykinase (PEPCK) is the key enzyme to initiate the gluconeogenic pathway in vertebrates, yeast, plants and most bacteria. Nucleotide specificity divided all PEPCK into two groups. All eukaryotic and most archaeal PEPCK are GTP-dependent. Bacterial and fungal PEPCK can be either ATP-or GTP-specific but all plant PEPCK are ATP specific [1]. Amino acid sequence alignment of PEPCK enzymes shows that the nucleotide binding sites are somewhat conserved within each class with few exceptions that do not have any clear ATP- or GTP-specific binding motif. Although the active site residues are mostly conserved in all PEPCK, not much significant sequence homology persists between ATP and GTP dependent PEPCK enzymes. There is only one planctomycetes PEPCK enzyme (from Cadidatus Kuenenia stuttgartiensis) that shows sequence homology with both ATP-and GTP-dependent PEPCK [1]. ATP-utilizing PEPCKs are monomers or oligomers of identical subunits found in certain bacteria, yeast, trypanosomatids, and plants, while GTP-utilizing PEPCK are mainly monomers found in animals and some bacteria. Both require divalent cations for activity, such as magnesium or manganese. One cation interacts with the enzyme at metal binding site-1 to elicit activation, while the second cation interacts at metal binding site-2 to serve as a metal-nucleotide substrate.

Natural products comprising plants, animals and minerals have been the basis for treatment of human diseases in traditional medicine by indigenous peoples, throughout the world and across time, is a well documented practice [2], [3], [4]. At the same time molecular modeling and docking have recently been introduced and gained attention among medicinal chemists [5]. It is believed that most of the chemotherapeutic agents directly or indirectly alter enzyme systems of helminth parasites mainly PEPCK [6], [7] and serves as a good antihelminthic agents. A limited choice of safe and efficacious drugs is currently available for the 
treatment of schistosomiasis, helminthiasis and most intestinal protozoa infections. However, none of the compounds covers the whole parasite spectrum and they were mostly developed decades ago [8], [9], [10]. The main anthelminthic drugs are praziquantel for the treatment of trematodes and cestodes, albendazole, mebendazole (both benzimidazol carbamates), levamisole and pyrantel pamoate for intestinal nematodes and ivermectin for $S$. stercoralis and lymphatic filariasis.

The $S$. japonicum worms are yellow or yellow-brown. The males of this species are slightly larger than the other Schistosomes and they measure $\sim 1.2 \mathrm{~cm}$ by $0.5 \mathrm{~mm}$. The females measure $2 \mathrm{~cm}$ by $0.4 \mathrm{~mm}$. The adult worms are longer and narrower than the related S. mansoni worms. Schistosoma japonicum is the only human blood fluke that occurs in China. It is the cause of Schistosomiasis japonica, a disease that still remains a significant health problem especially in lake and marshland regions. Schistosomiasis is an infection caused mainly by three schistosome species; Schistosoma mansoni, Schistosoma japonicum and Schistosoma haematobium. S. japonicum being the most infectious of the three species [11].

PEPCK is an important enzyme of functional significance in helminth parasites which catalyses a reverse reaction that forms oxaloacetic acid from phosphoenolpuruvate (PEP) rather than PEP formation from oxaloacetate as in mammals [12]. This branch point differences of energy transducing systems between helminth parasites and their hosts has given some indication that this pathways are quite different from those of the mammalian host which can be used in finding inhibitors that affect the parasite's metabolic pathways selectively [13], [14]. Because of its functional difference between the parasite and the host, it supports the belief that PEPCK could be a possible target for chemotherapeutic agents [15]. However, PEPCK has been isolated and characterized from only some selected helminth species, such as: Hymenolepis diminuta, the rodent dwarf tapeworm; Fasciola hepatica; Ascaris suum; Moniezia expansa, a cestode parasite of sheep; and Moniliformis dubius [16], [17], [18], [19], [20]. Wu et al. [21] have suggested the involvement of PEPCK (PEPCK: unigene 17356) in host-parasite interaction.

In the absence of experimental structures, the present study was carried out to use the computational methods to predict 3D protein structure of PEPCK of $S$. japonicum to provide insight into the structure and function of the enzyme.

\section{METHODOLOGY}

\subsection{Sequence retrieval and 3D model building}

The sequence of the PEPCK enzyme of Schistosoma japonicum was retrieved from NCBI database bearing Genebank ID AAW25103.1. Then with this query sequence a BLAST [22] search was performed against protein data bank to retrieve the corresponding template for the PEPCK enzyme with high similarity. The model was built by homology modeling and for this MODELLER [23] program was used. The MODELLER program uses an automated approach to comparative protein structure modeling by satisfaction of spatial restraints [24]. The template used in the present study was rat cytosolic PEPCK (PDB: 3DT7 Chain: A) in complex with beta-sulfopyruvate and GTP with experimental X-RAY Resolution 1.50.

\subsection{Model validation}

The MODELLER generated 3D structure of PEPCK was further verified by PROCHECK [25]. The PROCHECK program provides the information about the stereo chemical quality of a given protein structure. The PROCHECK was used to generate Ramachandran plot and the quality of the structure was computed in terms of $\%$ of residues in favorable regions, $\%$ of non Proline, Glycine residues etc. The secondary structure prediction was carried out using Swiss 3 D protein assessment server, a highly accurate method for protein secondary structure prediction. The quality of structure was also accessed by using ERRAT [26]. ERRAT is a protein structure verification algorithm that is especially well-suited for evaluating the progress of crystallographic model building and refinement. Search for Conserved Domains (CD) within a modeled protein was carried out using NCBI source (http://www.ncbi.nlm.nih.gov/Structure/cdd/wrpsb.cgi).

\subsection{ProQ - Protein Quality Predictor}

ProQ is a neural network based predictor that based on a number of structural features predicts the quality of a protein model. ProQ is optimized to find correct models in contrast to other methods which are optimized to find native structures. Two quality measures are generally considered which are predicited LGscore and MaxSub [27].

\subsection{Active site Prediction and Molecular Docking}

Sites of binding in proteins usually lie in cavities or on the polar surface. The size and shape of protein surface dictates the three-dimensional geometry of ligand that must binds due to intra atomic forces between them. The binding of a ligand typically serves as a mechanism for chemical modification or conformational changes of protein. Phosphoenolpyruvate carboxykinase (PEPCK), a carboxylase enzyme in nature (GTP or 
ATP dependent) is present in all living organisms. In the present study GTP binding site was determined and molecular docking was performed to evaluate GTP interaction with PEPCK modeled structure.

Molecular docking was performed with GTP using Molegro Virtual Docker 2010.4.0 (MDV2010.4.0). Molegro Virtual Docker software is best suited for all aspects of the docking process including preparation of the molecules to determination of the potential binding sites of the target protein, and prediction of the binding modes of the ligands [28]. This software is based on a new heuristic search algorithm that combines differential evolution with cavity prediction algorithm [29]. The binding site was detected by the docking software itself.

The docking scoring function, Escore, is defined by the following energy terms:

$E_{\text {score }}=E_{\text {inter }}+E_{\text {intra }}$

Where, $E$ inter is the ligand-protein interaction energy:

$$
E_{\text {inter }}=\Sigma_{i \in \text { ligand }} \sum_{j \in \text { protein }}\left[E_{\mathrm{PLP}}\left(r_{i j}\right)+332.0 \frac{q_{i} q_{j}}{4 r_{i j}^{2}}\right]
$$

$E$ intra is the internal energy of the ligand:

$$
E_{\text {intra }}=\sum_{i \in l i g a n d} \sum_{j \in l i g a n d} E_{\mathrm{PLP}}\left(r_{i j}\right)+\sum_{\text {flexible bonds }} A\left[1-\cos \left(m * \theta-\theta_{0}\right)\right]+E_{\text {clash }}
$$

The search space of the simulation exploited in the docking studies was defined as a subset region of $15.0 \AA$ around the active site cleft. The overall geometry of GTP was optimized by the Powell Method [30] using Tripos Force Field [31]. 1000 iterations were given with a convergence criterion of $0.061 \mathrm{kcal} / \mathrm{mol} \AA$. Charge distributions were calculated by Gasteiger-Marsilli method [32]. Proper bonds, bond orders, hybridization and charges were assigned using the Molegro Virtual Docker (MVD - 2010, 4.0). Ten runs were performed and five poses returned. All the other docking parameters were set as default. The license file of fully functional MVD software was requested from Rene Thomsen (http://www.molegro.com/).

\subsection{Function prediction of modeled PEPCK using 3d2GO Server}

$3 \mathrm{~d} 2 \mathrm{GO}$ server was used to predict the possible function of the predicted model using sequence and structure in the reference of Gene Ontology. It predicts the function of the protein using sources of information like overall topological similarity to structures with known function, geometric and residue similarity of predicted functional sites to regions of known structures and sequence homology to functionally annotated sequences [33].

\subsection{Homology modeling}

\section{RESULT AND DISCUSSION}

Homology modeling is a very effective method of generating the 3D structure of a protein for which experimental (X-rays crystallographic or NMR) structure is not available but the crystal structure of homologue is available. Then by making use of this template the 3D structure of the protein of interest was generated through homology modeling based method [34], [35], [36].

For homology modeling of PEPCK from $S$. japonicum, the structure of rat cytosolic PEPCK in complex with beta- 2 sulfopyruvate and GTP (PDB ID: 3DT7) was selected as a template. Multiple sequence alignment (Fig.1) was performed on Clustal-W using GONNET matrix and subjected to MODELLER. There were total 622 amino acid residues in template structure whereas; query sequence contains 624 amino acids. Total ten models were generated against the template 3DT7. The best ranked model (Fig. 2) was then subjected for evaluation to check the quality of the generated model. There was more than $62 \%$ sequence identity with $E$ value $0.00 \mathrm{e}^{-1}$ in query and template sequences. Then MODELLER was used to generate the three dimensional structure of PEPCK. It is found that template structure contains different ligand which includes GTP800, MN700, MN701, MN703 and NA 100. In case of modeled protein also all these ligands were incorporated during model generation. GTP800 interacts, within a radius of 3 Angstrom, with the following ions: MN700, MN701 and MN703.

\subsection{Structure validation}

To obtain an accurate homology model, it is very important that appropriate steps are built into the process to assess the quality of the model. Therefore, accuracy of the predicted models was subjected through a series of tests [34]. To verify the quality of predicted model structures, the coordinates of predicted structures was submitted into the ERRAT Protein Verification Server. The overall quality factor was obtained as 95.33 which are reasonable for the modeled PEPCK (Fig. 3). ERRAT plot assesses the spatial arrangement of different atoms in the protein models. It is a sensitive technique, which is good for identifying incorrectly-folded regions in preliminary protein models. ERRAT is a so-called "overall quality factor" for non-bonded atomic interactions, and higher scores mean better quality [34]. 
In order to assess the reliability of the modeled structure of PEPCK, we calculated the root mean square deviation (RMSD) by superimposing it on the template structure (Fig. 4). We have compared the protein structures using root mean square deviation (RMSD) which measures the difference between C $\alpha$-atom positions between two proteins. The smaller the deviation, the better is spatially equivalent of two proteins. Superimposition of protein structures was done using Chimera program (https://www.cgl.ucsf.edu/chimera/). The value of RMSD of two superimposed protein structures was found to be $0.215 \AA$. The superimposed structure clearly showed to build from the same template.

The Z-score QMEAN [37] value for modeled structure was 0.22 (Fig. 5). This score is composed of a linear combination of 6 terms, where the pseudo-energy of the contributing terms is given with respect to the scores obtained for high-resolution experimental structures of similar size solved by X-ray crystallography. WHATIF Z-score, obtained by assessing the 3D structure through a series of measurements of structures crystallized by X-ray and NMR, was - 11.2 (Fig. 6). To be acceptable, this value must be below - 6 [38].

The ability to segregate between correct and incorrect models of protein is of the great importance for protein structure prediction methods. ProQ, a neural network- based method to predict the quality of a protein model that extracts structural features, such as frequency of atom-atom contacts, and predicts the quality of a model, as measured either by LGscore or MaxSub. ProQ-LG denotes a network predicting LGscore and ProQMX denotes networks predicting MaxSub. The overall quality factor of modeled protein was also assessed by ProQ software which showed extremely good structure based on predicted LG score 6.948 and Maxsub score 0.579 (Table 1).

The validation of the model was also carried out using Ramachandran plot calculations computed with the PROCHECK program [25]. The $\Phi$ and $\Psi$ distributions of the Ramachandran plots of non-Glycine, nonProline residues are summarized in Fig. 7. Altogether $99.2 \%$ of the residues was in favored and allowed regions (Fig. 7). The percentage of amino acids in core, allowed, general and disallowed regions were 97.6\%, 0.9\%, $0.6 \%$ and $0.8 \%$ respectively. A global Z-score value of -0.43 obtained from the Ramachandran plot. A Z-score value greater than -2.0 is commonly associated with a high-quality model [38].

\subsection{Characterization of Model Structure}

Structurally, when the model protein was submitted to the EMBL-EBI database, it generated the secondary structure along with many other data [25]. The model structure of S. japonicum PEPCK consists of 30 strands, 17 helices, 8 beta hairpins, 6 number of 3, 10 helices (Fig. 8). Topological secondary structure representation of 624 amino acid residues of PEPCK model protein of S. japonicum is shown in Fig. 9. Predicted solvent accessibility composition (core/surface ratio) for the modeled protein showed residues exposed with more than $16 \%$ of their surface is $43.93 \%$ and all other residues occupy $56.07 \%$ in protein structure (http://www.predictprotein.org). The theoretical isoelectric point and molecular weight was also calculated from the Expasy tool [39] and found to be 6.35 and 70334 (Da) respectively. Earlier studies by Asahi et al. [40] and Knudsen et al. [41] showed that the MW of S. mansoni PEPCK is $62 \mathrm{kDa}$ and $70.4 \mathrm{kDa}$ respectively. Similarly, the MW of Ascaris suum has also been found to be $83 \mathrm{kDa}$ [42].

EMBOSS Pepinfo creates a variety of plots that display different amino acid properties, such as hydropathy or charged residues, and their position in the sequence. In the present study amino acid sequences of model protein was submitted to Pepinfo and hydropathy plot was constructed (Fig. 10). The hydropathy plot is used to find clusters of hydrophobic amino acids, which could indicate that the polypeptide in question is a transmembrane protein. A transmembrane protein has hydrophilic parts which protrude out on either side of the cellular membrane, and a hydrophobic centre which lies within the membrane [43].

Using 3D Ligand site-Ligand binding site prediction server (http://www.sbg.bio.ic.ac.uk/) probability of amino acid residue forming a binding cavity is predicted which is shown in Fig. 11). 3DLigandSite is a web server for the prediction of ligand-binding sites [44]. It is based upon successful manual methods used in the eighth round of the critical assessment of techniques for protein Structure Prediction (CASP8).

\subsection{Docking studies}

PEPCK was modeled computationally and its active sites were also predicted using inbuilt program in Molegro virtual docker software. All the eukaryotic mammalian and most archaeal PEPCKs are GTP-specific. Therefore, in the present study docking was perform with GTP in the binding site of modeled protein (PEPCK) to predict the functional aspect of modeled protein. The approximate location of active site, GTP binding site, metal binding site and substrate binding sites are shown in Fig. 12. Docking result showed that GTP binding site are conserved in model structure with similar binding affinity with template protein. The amino acid present in the GTP binding site are Pro 288, Ser 289, Ala 290, Cys 291, Gly 292, Lys 293, Thr 294, Asn 295, Pro 340, Arg 410, Thr 294, Asn 295, Pro 340, Arg 410, Arg 441, Trp 520, Phe 521, Phe 534 and Asn 537. In case of template protein the docking score, re-rank score and RMSD value were $-250.45,-153.23$ and 0.9015 , whereas in case of modeled protein it was $-265.45,-199.05$ and 0.5351 respectively, which showed modeled protein accommodate GTP more strongly in its active site as compared to template protein. The amino acids involved during H-bond 
formation in modeled protein are shown in Fig. 13. In addition, we observed that the same amino acids in both template and modeled protein were involved in hydrogen-bond formation with GTP during docking procedures. 3.5 Domain prediction of PEPCK modeled protein

Search for Conserved Domains (CD) within a modeled protein was carried out using NCBI source (http://www.ncbi.nlm.nih.gov/Structure/cdd/wrpsb.cgi). The result showed that the modeled protein consists of two conserved domains. Domain 1: Superfamily- PEP carboxykinase-like (E-value 5.26e-150); Family: PEP carboxykinase C-terminal domain (E value 5.09e-10); amino acid range: 263-623. Domain 2: Superfamily-PEP carboxykinase N-terminal domain (E value: 1.1e-97); Family: PEP carboxykinase N-terminal domain (1.29e07), amino acid range: 13-262. The results are sorted from lowest E-value to highest E-value. Strong classifications have a low E-value. Ambiguous hits are not shown on the domain architecture. The family level classification is conditional on the domain being a member of the specified superfamily. There is a possibility that the selected domain is a member of a sub-family for which no structure has yet been solved. In this case the family E-value will likely to be $>0.01$ [45]. The related conserved domain (cd00819: PEPCK_GTP) and substrate binding domain (c100270: PEPCK HprK Superfamily) were also determined and compared with similar domains from others gene present in NCBI gene bank which phylogeny cluster is shown in Fig. 14. $\mathrm{HprK} / \mathrm{P}$, the bi-functional histidine-containing protein kinase/phosphatase controls the phosphorylation state of the phosphocarrier protein, HPr and regulates the utilization of carbon sources.

\subsection{Function prediction of modeled protein}

The 3d2GO server (http://www.sbg.bio.ic.ac.uk/phyre/ pfd/) was used to predict the function of the protein model. This uses several methods of function prediction, using sequence and structure, to predict Gene Ontology (GO) terms for the protein. Various GO terms, their description and the confidence have been listed in Table 2. Confidence ranges from 0 to 1 , with 1 being the most confident prediction. Result show that the predicted protein has different functions like Carboxy-lyase activity, Phosphoenol pyruvate carboxikinase activity, Carbon-carbon lyase activity, ion binding, metal ion binding, Carboxylic acid binding, GTP binding activities with high confidence.

\section{FIGURES AND TABLES}

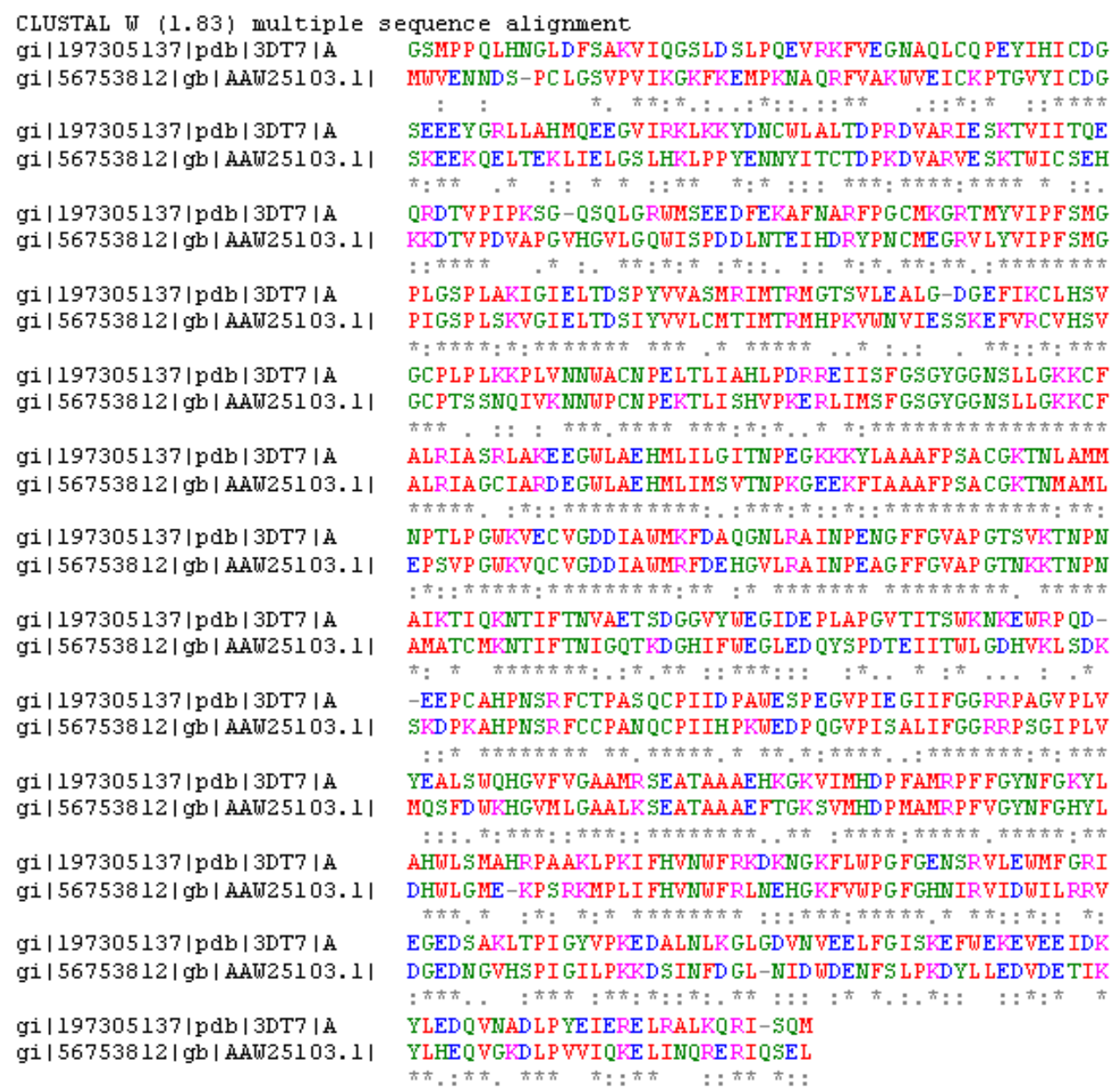

Figure. 1 The pair wise sequences alignment of 3DT7 (template) and S. japonicum PEPCK sequence (query). 


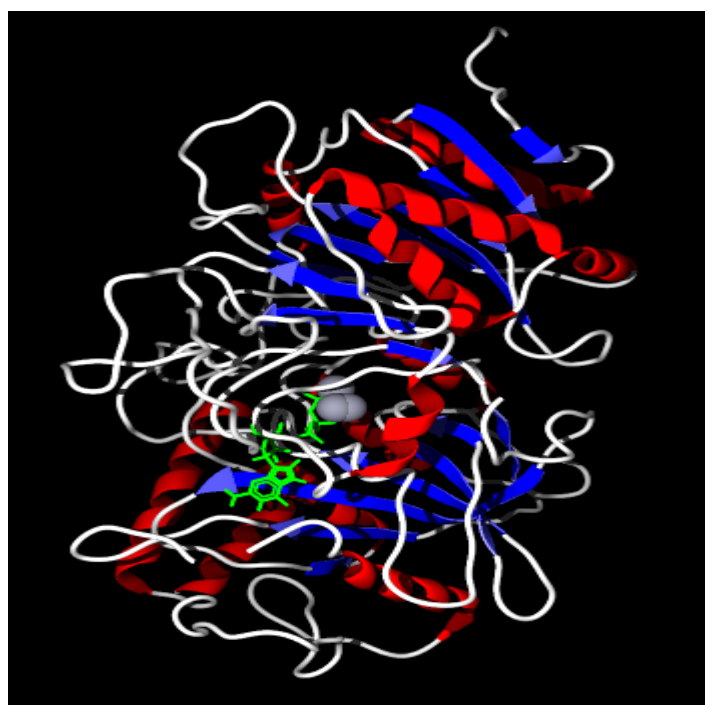

Figure. 2 3D modeled structure of the S. japonicum Phosphoenolpyruvate carboxykinase (PEPCK).
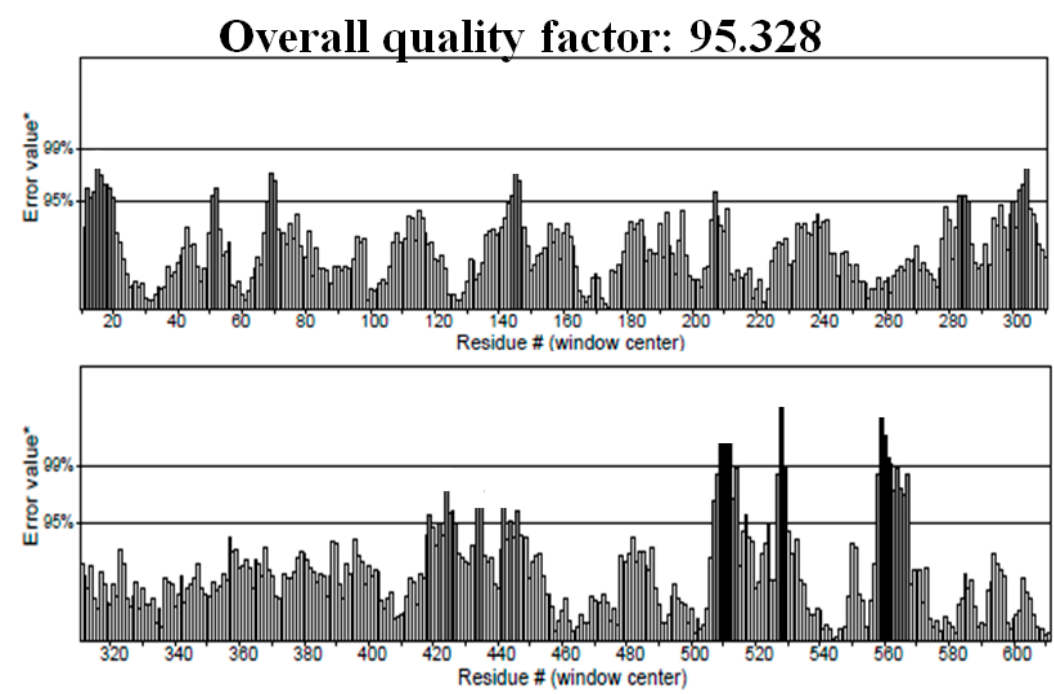

Figure. 3 Overall quality factor or ERRAT score of PEPCK modeled protein. Black bars shows the misfolded region located distantly from the active site, gray bars demonstrate the error region between $95 \%$ and $99 \%$, white bars indicate the region having less error rate for protein folding. Good high resolution structure generally produce values around $95 \%$ or higher. For lower resolution ( 2.5 to $3 \AA)$ the average over all quality factors is around 91\%".

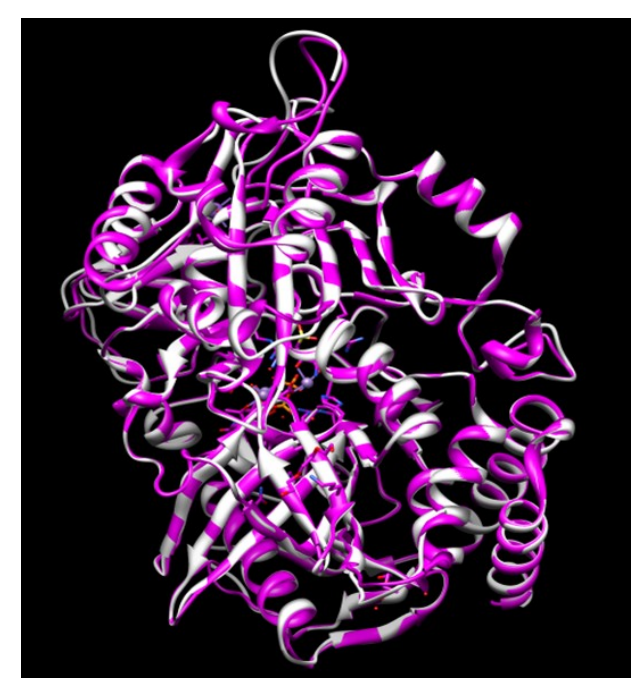

Figure. 4 Superimposition of the modeled protein and template protein using CHIMERA, software. 


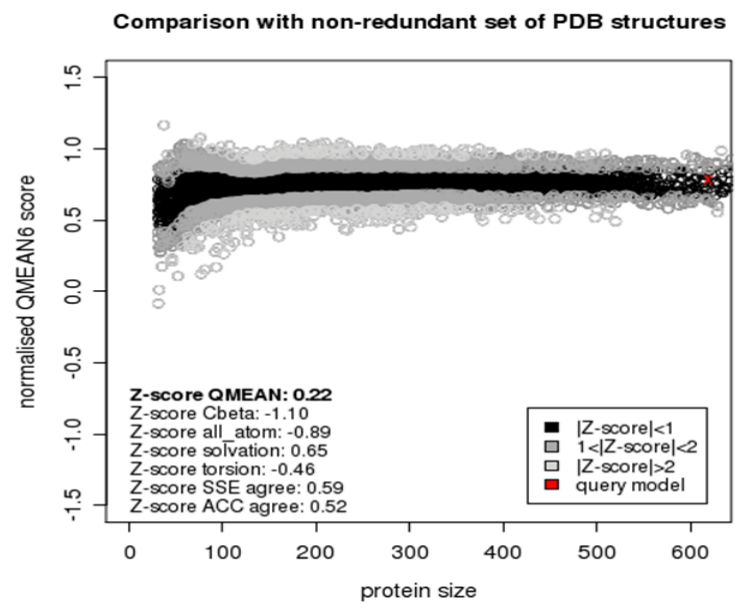

Figure. 5 QMEAN6 plot normalized shows the standard deviation of modeled protein.

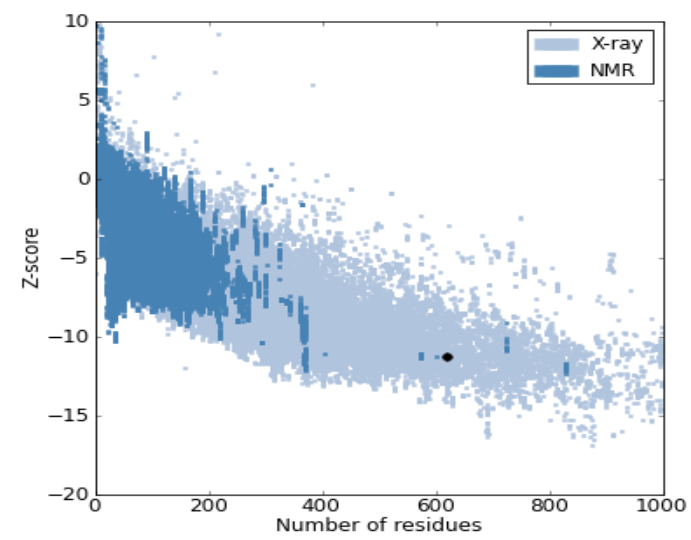

Figure. 6 ProSA-web z-scores of model (black arrow) in relation to all protein chains in PDB determined by Xray crystallography (light blue) or NMR spectroscopy (dark blue) with respect to their length. The plot shows only chains with less than 1000 residues and a $\mathrm{z}$-score $>10$.

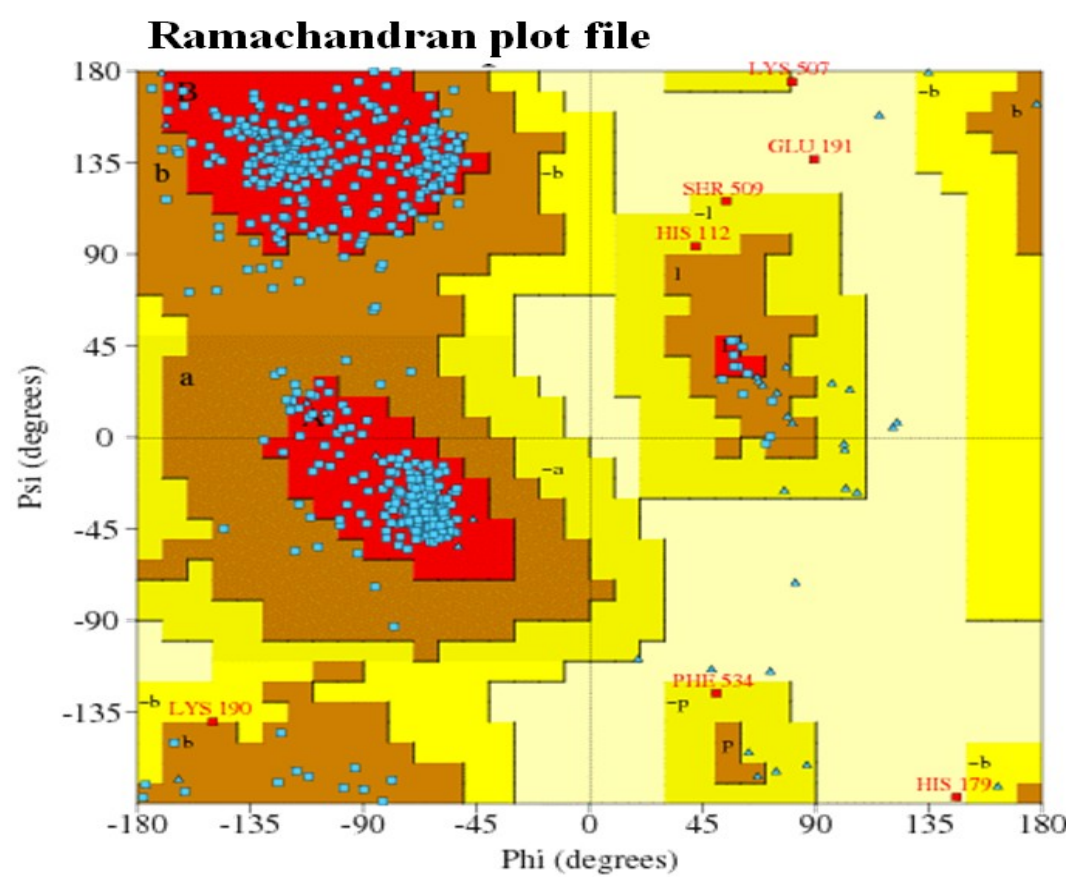

Figure. 7 Ramachandran plot showing the distribution of amino acids residues in a modeled PEPCK structure. 


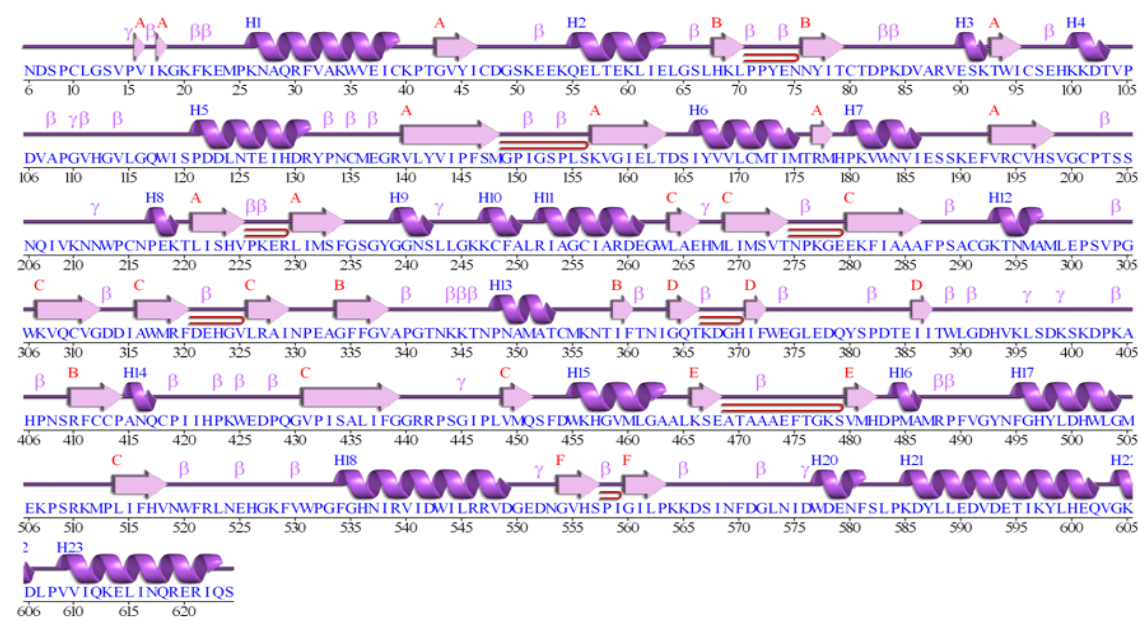

Figure. 8 Prediction of secondary structure of model, phosphoenolpyruvate carboxykinase using Swiss Protein $3 \mathrm{D}$ assessment server.

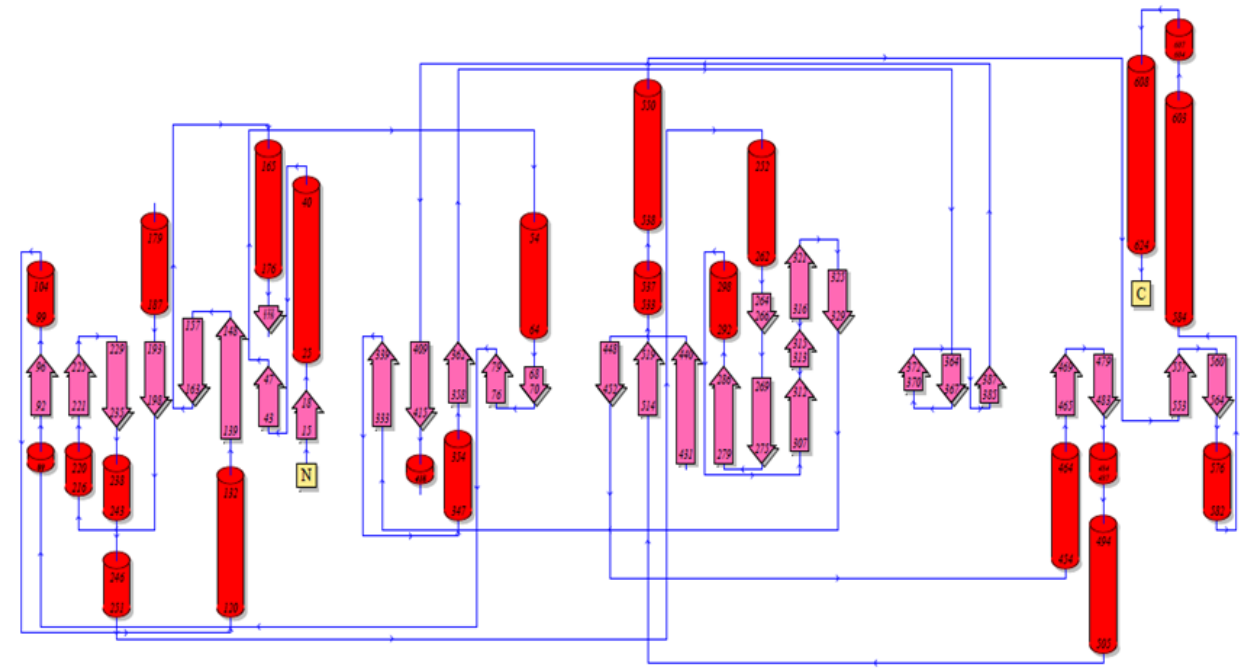

Figure. 9 Topological secondary structure representation of 626 amino acid residues in PEPCK model protein of S. joponicum.

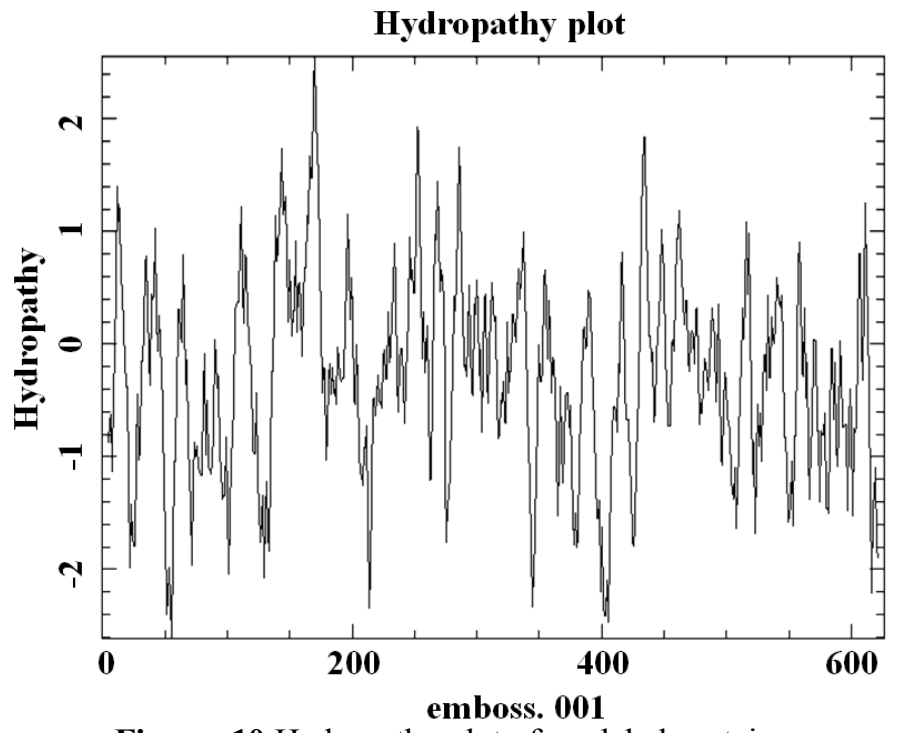

Figure. 10 Hydropathy plot of modeled protein. 


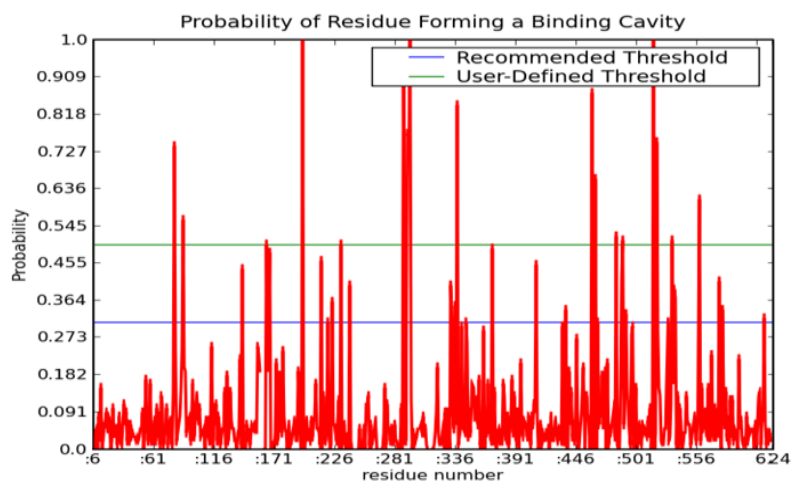

Figure. 11 Prediction of ligand-binding sites using 3DLigandSite web server.

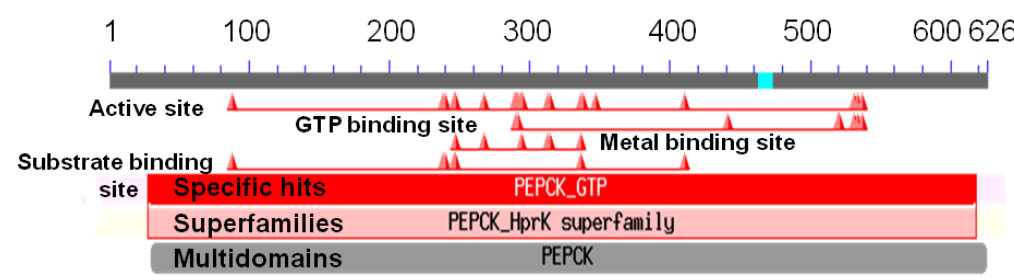

Figure. 12 Prediction of conserved domain in PEPCK structure.
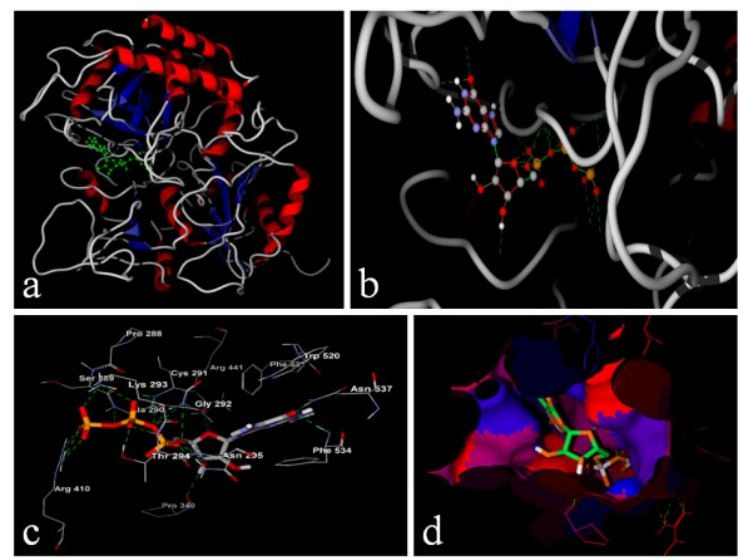

Figure. 13 Docking structure of GTP with modeled protein in GTP binding site. (a) Showing 3D structure of PEPCK in secondary structure format. (b) Docking view in secondary structure format. (c) Interaction of amino acid with GTP. (d) Showing GTP fitting in the GTP binding domain.
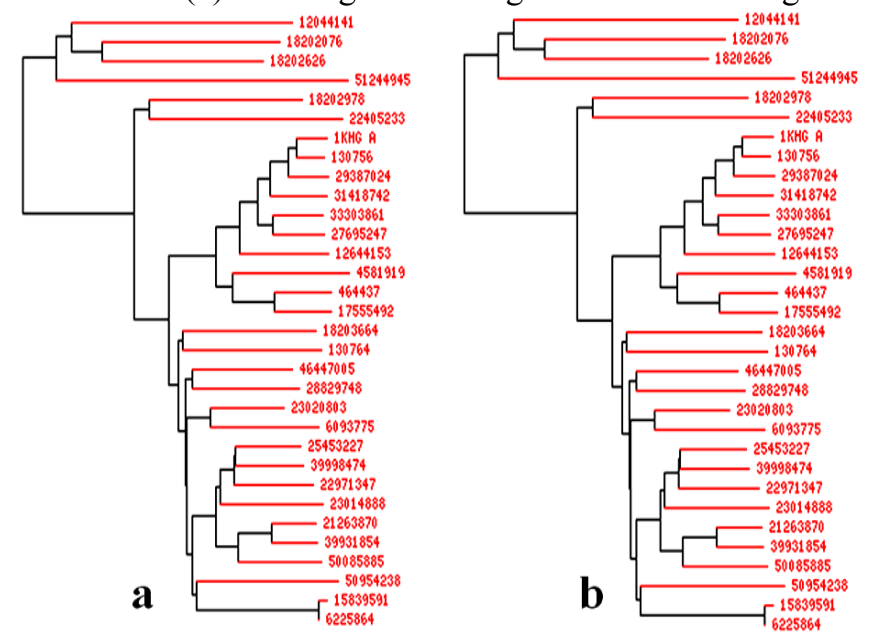

Figure. 14 Cluster analysis tree showing the relationship between substrate binding conserved domain and GTP binding conserved domain of related gene sequences submitted in Gene bank. (a) Substrate binding conserved 
domain and (b) GTP binding conserved domain. The number on the branch showing the GI number of related sequences submitted in NCBI gene bank.

Table 1. ProQ based evaluation of the quality of model protein. Two quality measures are used,

\begin{tabular}{lc}
\multicolumn{2}{c}{ predicited LGscore and MaxSub. } \\
\hline Different ranges of quality & ProQ - Results \\
\hline LGscore $>1.5$ fairly good model & Predicted LGscore : 6.948 \\
LGscore $>2.5$ very good model & \\
LGscore $>4$ extremly good model & Predicted MaxSub : 0.579 \\
\hline MaxSub $>0.1$ fairly good model & \\
MaxSub $>0.5$ very good model & \\
MaxSub $>0.8$ extremly good model & \\
\hline
\end{tabular}

Table 2. Predicted function of modeled protein with their confidence limits.

\begin{tabular}{|c|c|c|}
\hline GO term & Description & Confidence limit \\
\hline GO: 0000166 & Nucleotide binding & 0.99 \\
\hline GO: 0005737 & Cytoplasm & 0.96 \\
\hline GO: 0016831 & Carboxy-lyase activity & 0.96 \\
\hline GO: 0004611 & Phosphoenol pyruvate carboxikinase activity & 0.96 \\
\hline GO: 0006094 & Gluconeogenesis & 0.96 \\
\hline GO: 0016829 & Lyase activity & 0.95 \\
\hline GO: 0017076 & Purine nucleotide binding & 0.95 \\
\hline GO: 0000287 & Magnesium ion binding & 0.93 \\
\hline GO: 0032561 & Guanyl ribonucleotide binding & 0.91 \\
\hline GO: 0016830 & Carbon-carbon lyase activity & 0.91 \\
\hline GO: 0032553 & Ribonucleotide binding & 0.91 \\
\hline GO: 0032555 & Purine ribonucleotide binding & 0.91 \\
\hline GO: 0005634 & Nucleus & 0.90 \\
\hline GO: 0005525 & GTP binding & 0.90 \\
\hline GO: 0030145 & Maganese ion binding & 0.90 \\
\hline GO: 0046327 & Glycerol biosynthesis from pyruvate & 0.90 \\
\hline GO: 0004613 & Phosphoenol pyruvate carboxikinase activity (GTP) & 0.90 \\
\hline GO: 0032868 & Response to insulin stimulus & 0.90 \\
\hline GO: 0031406 & Carboxylic acid binding & 0.90 \\
\hline GO: 0042593 & Glucose homeostasis & 0.90 \\
\hline GO: 0005813 & Centrosome & 0.90 \\
\hline GO: 0006006 & Glucose metabolic process & 0.90 \\
\hline GO: 0006629 & Lipid metabolic process & 0.90 \\
\hline GO: 0046914 & Transition metal ion binding & 0.88 \\
\hline GO: 0046872 & Metal ion binding & 0.88 \\
\hline GO: 0043167 & Ion binding & 0.88 \\
\hline GO: 0043169 & Cation binding & 0.87 \\
\hline GO: 0003824 & Catalytic activity & 0.76 \\
\hline
\end{tabular}

\section{CONCLUSION}

In the present study homology modeling and function prediction of PEPCK was performed. The predicted model was validated with different program including Procheck, ERRAT, ProQ, Expasy tools etc. Ramachandran plot for modeled protein showed more than $99 \%$ residues are in allowed and additionally allowed regions. The Carboxy-lyase activity, Phosphoenol pyruvate carboxykinase activity, Carbon-carbon lyase activity, ion binding, metal ion binding, Carboxylic acid binding, GTP binding activities were conserved as important functional site of the model with high confidence. Docking study showed modeled protein is able to dock with GTP more strongly in its active site as compared to template protein. Based on the results of present study it can be suggested that this model is reliable and could be useful for understanding the binding mode and for the design of new PEPCK inhibitors. Since, the inhibition of PEPCK is predicted to be a major target for antihelminthic drugs due to its functional difference between the parasites and the hosts. These findings are the subject to experimental verification and application for the finding of new chemotherapeutic agent(s) to combat schistosomiasis, helminthiasis and most intestinal protozoa infections problem.

\section{Acknowledgements}

We acknowledge the University Grant Commission, New Delhi (India) for providing Research fellowship in science for meritorious students (F-4-1/2006(BSR)/5-120/2007(BSR).

\section{Compliance with ethics guidelines}

Conflict of Interest

All authors declare that they have no conflict of interest.

Human and animal rights, informed consent 
This article does not contain any studies with human or animal subjects performed by the any of the authors.

\section{REFERENCES:}

[1]. Fukuda W, Fukui T, Atomi H, and Imanaka T, First Characterization of an Archaeal GTP-Dependent Phosphoenolpyruvate Carboxykinase from the Hyperthermophilic Archaeon Thermococcus kodakaraensis KOD1, J Bacteriol, 186: 2004, 4620-4627.

[2]. Prasad, S B, Verma A K, Rosangkima G, Brahma B, Rongpi T, Amenla, and Arjun J, Antitumor activity of Mylabris cichorii extracts against murine ascites Dalton's lymphoma, J Pharm Res, 3: 2010, 3006-3009.

[3]. Verma A K, and Prasad S B, Bioactive component, cantharidin from Mylabris cichorii and its antitumor activity against Ehrlich ascites carcinoma, Cell Biol Toxicol, 28: 2012, 133-147.

[4]. Brahma B, Prasad S B, Verma A K, and Rosangkima G, Study on the antitumor efficacy of some select medicinal plants of Assam against murine ascites Dalton's lymphoma, Pharmacologyonline, 3: 2011, 155-168.

[5]. Basu Baul T S, Paul A, Pellerito L, Scopelliti M, Singh P, Verma P, Duthie A, de Vos D, and Tiekink E R T, Dibutyltin (IV) complexes containing arylazobenzoate ligands: Chemistry, in vitro cytotoxic effects on human tumor cell lines and mode of interaction with some enzymes, Invest New Drugs, 29: 2011, 285-299.

[6]. Roy B, and Swargiary A, Anthelmintic efficacy of ethanolic shoot extract of Alpinia nigra on tegumental enzymes of Fasciolopsis buski, a giant intestinal parasite, $J$ Parasit Dis, 33: 2009, 48-53

[7]. Vokral I, Krizova V, Lamka J, Kubicek V, Szotakova B, Varady B, Nobilis M, and Skalova L, Effect of flubendazole on biotransformation enzymes activities in Haemonchus contortus, Open Parasitol J, 4: 2010, 24-28.

[8]. WHO, Prevention and control of schistosomiasis and soil-transmitted helminthiasis: report of a WHO expert committee, WHO Technical Report Series, 912: 2002, 1-57.

[9]. Utzinger J, and J Keiser, Schistosomiasis and soil-transmitted helminthiasis: common drugs for treatment and control, Expert Opin Pharmacother, 5: 2004, 263-285

[10]. Farthing M J, Treatment options for the eradication of intestinal protozoa, Nat Clin Pract Gastroenterol Hepatol, 3: 2006, 436-445.

[11]. Jia T W, Zhou X N, Wang X H, Utzinger J, Steinmann P, and Wu X H, Assessment of the age-specific disability weight of chronic Schistosomiasis japonica, Bull World Health Organ, 85: 2007, 458-465.

[12]. Barrett J, Forty years of helminth biochemistry, Parasitology, 136: 2009,633-1642

[13]. Omura S, Miyadera H, Ui H, Shiomi Z, Yamaguchi Y, Masuma R, Nagamitsu T, Takano D, Sunazuka T, Harder A, Kolbl H, Namikoshii M, Miyoshi H, Sakamoto K, and Kita K, An anthelmintic compound, nafuredin, shows selective inhibition of complex I in helminth mitochondria. Proc Nat Acad Sci, 98: 2001, 60-62.

[14]. Mansour T E, Chemotherapeutic targets in parasites contemporary strategies. (Cambridge University Press, Cambridge, 2002).

[15]. Geary T G, and Winterrowd C A, Alexander-Bowman S J, Favreau M A, Nulf S C, Klein R D, Ascaris suum: cloning of a cDNA encoding phosphoenolpyruvate carboxykinase, Exp Parasitol, 77: 1993, 155-161.

[16]. Behm C A, and Bryant C, Studies of regulatory metabolism in Moniezia expansa: The role of phosphoenolpyruvate carboxykinase. Int J Parasitol, 5: 1975, 347--354.

[17]. Behm, C A, and Bryant C, Phosphoenolpyruvate carboxykinase from Fasciola hepatica. Int J Parasitol, 12: 1982, 271-278.

[18]. Cornish R A, Wilkes J, and Mettrick D F, A study of phosphoenolpyruvate carboxykinase from Moniliformis dubius (acanthocephala). Mol Biochem Parasitol, 2: 1981, 151-166.

[19]. Wilkes J, Cornish R A, and Mettrick D F, Purification and properties of phosphoenolpyruvate carboxykinase from Ascaris suum, Int J Parasitol, 12: 1982, 163-171.

[20]. Rohrer S P, Saz H J, and Nowak T, Purification and characterization of phosphoenolpyruvate carboxykinase from the parasitic helminth Ascaris suum, J Biol Chem, 261: 1986, 13049-13055.

[21]. Wu X, Fu Yan, Yang D, Zhang R, Zheng W, Nie H, Xie Y, Yan N, Hao G, Gu X, Wang S, Peng X, and GYang G, Detailed transcriptome description of the neglected cestode Taenia multiceps, PLOS ONE, 9: 2012, 1-11.

[22]. Altschul S F, Gish W, Miller W, Myers E W, and Lipman D J, Basic local alignment search tool. J Mol Biol, 215: 1990, 403-410.

[23]. Sali A, and Blundell T L, Comparative protein modeling by satisfaction of spatial restraints. J Mol Biol, 234: 1993, 779-815.

[24]. Eswar N, Eramian D, Webb B, Shen M, and Sali A, Protein structure modeling with Modeller, Methods Mol Biol, 426: 2008, 145159.

[25]. Laskowski R A, MacArthur M W, Moss D S, and Thornton J M, PROCHECK: a program to check the stereo chemical quality of protein structures, J Appl Cryst, 26: 1998, 283-291.

[26]. Colovos V C, and Yeates T O, Verification of protein structures: Patterns of none bonded atomic interactions, Protein Sci, 2: 1993, 1511-1519.

[27]. Wallner B, and Elofsson A, Can correct protein models be identified? Protein Sci, 12: 2003, 1073-1086

[28]. Avupati V R, Yejella R P, Akula A, Guntuku G S, Doddi B R, Vutla V R, Anagani S R, Adimulam L S, and Vyricharla A K, Synthesis, characterization and biological evaluation of some novel 2, 4-thiazolidinediones as potential cytotoxic, antimicrobial and antihyperglycemic agents. Bioorg Med Chem Lett, 22: 6442-6450. Thomsen R, Christensen M H (2006). MolDock: a new technique for high -accuracy docking. J Med Chem, 49: 2012, 3315-3321.

[29]. Thomsen R, and Christensen M H, MolDock: a new technique for high -accuracy docking, J Med Chem, 49: 2006, 3315-3321.

[30]. Fletcher R, and Powell M J D, A rapidly convergent descent method for minimization, Comp J, 6: 1963, 163-168.

[31]. Clark M, Cramer R D, and Van Obdenbosch N, The tripos force field, J Computation Chem, 10: 1989, 982-1012.

[32]. Gasteiger J, and Marsili M, Iterative partial equalization of orbital electro negativity a rapid access to atomic charges, Tetrahedron, 36: 1980, 3219-3228.

[33]. Moll M, and Kavraki L E, Matching of Structural Motifs Using Hashing on Residue Labels and Geometric Filtering for Protein Function Prediction, Proc. The Seventh Annual International Conference on Computational Systems, Bioinformatics. (CSB2008), Stanford, CA, 2008, 123-28.

[34]. Senthil K M, Selvam K, and Singaravel R, Homology modeling of a unique extracelluar glutaminase free L-asparaginase from novel marine Actinomycetes, Int J Res Biotechnol Biochem, 2: 2012, 1-12.

[35]. Verma A K, Swargiary A, Prasad S B, and Arjun J, Homology modeling of phosphoenolpyruvate carboxykinase of Ascaris suum, $J$ Pharm Res, 5: 2012, 1248-1255.

[36]. Swargiary A, Verma A K, and Sarma K, Homology modeling and docking studies of Phosphoenolpyruvate carboxykinase in Schistosoma mansoni, Med Chem Res, 2012, DOI 10.1007/s00044-012-0289-2

[37]. Benkert P, Biasini M, and Schwede T, Toward the estimation of the absolute quality of individual protein structure models. Bioinformatics, 27: 2011, 343-350.

[38]. Mendez-Cuesta C A, Mendez-Lucio O, and Castillo R, Homology modeling, docking and molecular dynamics of the Leishmania mexicana arginase: A description of the catalytic site useful for drug design, J Mol Graphic Mod, 38: 2012, 50-59. 
[39]. Gasteiger E, Gattiker A, Hoogland C, Ivanyi I, Appel R D and Bairoch A, ExPASy: the proteomics server for in-depth protein knowledge and analysis, Nucl Acids Res, 31: 2003, 3784-3788.

[40]. Asahi H, Osman A, Cook R M, LoVerde P T, and Stadecker M J, Schistosoma mansoni phosphoenolpyruvate carboxykinase, a novel egg antigen: immunological properties of the recombinant protein and identification of a T-cell epitope, Infect Immun, 68: 2000, 3385-3393.

[41]. Knudsen G M, Medzihradszky K F, Lim K C, Hansell E, and McKerrow J H, Proteomic analysis of Schistosoma mansoni cercarial secretions, Mol Cell Proteomics, 4: 2005, 1862-1875.

[42]. Rohrer S P, Saz H J, and Nowak T, Purification and characterization of phosphoenolpyruvate carboxykinase from the parasitic helminth Ascaris suum, J Biol Chem, 261: 1986, 13049-13055.

[43]. Kyte J, and Doolittle R F, A Simple Method for Displaying the Hydropathic Character of a Protein, J Mol Biol, 157: 1982, 105-132.

[44]. Wass M N, Kelley L A and Sternberg M J E, 3DLigandSite: predicting ligand-binding sites using similar structures, Nucleic Acids Res, 38: 2010, 469-473.

[45]. Marchler-Bauer A, Lu S, Anderson J B, Chitsaz F, Derbyshire M K, DeWeese-Scott C, Fong J H, Geer LY, Geer R C, Gonzales N R, Gwadz M, Hurwitz D I, Jackson J D, Ke Z, Lanczycki C J, Lu F, Marchler G H, Mullokandov M, Omelchenko M V, Robertson C L, Song J S, Thanki N, Yamashita R A, Zhang D, Zhang N, Zheng C, and Bryant S H, CDD: a Conserved Domain Database for the functional annotation of proteins, Nucleic Acids Res, 39: 2011, 225-229. 